\title{
COMPLEX DOUBLES OF BORDERED KLEIN SURFACES WITH MAXIMAL SYMMETRY
}

\author{
by COY L. MAY
}

(Received 5 August, 1989)

1. Introduction. A compact bordered Klein surface $X$ of algebraic genus $g \geq 2$ has maximal symmetry [6] if its automorphism group $A(X)$ is of order $12(g-1)$, the largest possible. The bordered surfaces with maximal symmetry are clearly of special interest and have been studied in several recent papers ([6] and [9] among others).

Associated with a bordered Klein surface $X$ in a natural way is its complex double $X_{c}$ [1], a classical Riemann surface of the same genus $g$. Suppose that $X$ has maximal symmetry. Then it is natural to ask how large the automorphism group of the complex double $X_{c}$ can be. Since the bordered surface $X$ is a very symmetrical object, then $X_{c}$ should also be very symmetrical. Indeed, it is easy to show that $X_{c}$ always has at least $24(g-1)$ automorphisms, and we originally expected that in several cases $X_{c}$ would have a larger automorphism group. Of course, the surface $X_{c}$ has at most $168(g-1)$ automorphisms (including the orientation-reversing ones); this is just twice the classical bound of Hurwitz.

Here we prove, however, that the order of the automorphism group of $X_{c}$ is $24(g-1)$, with a single exception. There is a unique Klein surface $Y$ (defined in $\$ 4)$ with maximal symmetry such that its complex double has $48(g-1)$ automorphisms. The surface $Y$ has genus two and topologically is a sphere with three holes. Our main result is the following.

THEOREM 1. Let $X$ be a bordered Klein surface with maximal symmetry of genus $g \geq 2$. If $X$ is not dianalytically equivalent to the surface $Y$ of genus 2 , then the automorphism group of the complex double $X_{c}$ is isomorphic to $C_{2} \times A(X)$.

2. NEC groups. Non-euclidean crystallographic (NEC) groups have been quite helpful in studying automorphism groups of Klein surfaces. Let $\mathscr{L}$ denote the group of automorphisms of the open upper half-plane $D$, and let $\mathscr{L}^{+}$denote the subgroup of index 2 consisting of the orientation-preserving automorphisms. An NEC group is a discrete subgroup $\Gamma$ of $\mathscr{L}$, and we shall assume that the quotient space $D / \Gamma$ is compact. An NEC group contained in $\mathscr{L}^{+}$is called a Fuchsian group. If $\Gamma$ is an NEC group containing orientation-reversing elements, then $\Gamma$ is called a proper NEC group. In this case $\Gamma$ has a canonical Fuchsian subgroup $\Gamma^{+}=\Gamma \cap \mathscr{L}^{+}$of index 2 .

Associated with the NEC group $\Gamma$ is its signature, which has the form

$$
\left(p ; \pm ;\left[m_{1}, \ldots, m_{r}\right] ;\left\{\left(n_{11}, \ldots, n_{1 s_{1}}\right), \ldots,\left(n_{k 1}, \ldots, n_{k s_{k}}\right)\right\}\right) \text {. }
$$

The quotient space $X=D / \Gamma$ is a compact surface with topological genus $p$ and $k$ holes. The surface is orientable if the + sign is used and non-orientable if the - sign is used. The integers $m_{1}, \ldots, m_{r}$, called the ordinary periods, are the ramification indices of the natural quotient mapping from $D$ to $X$ in fibers above interior points of $X$. The integers $n_{i 1}, \ldots, n_{i s_{i}}$, called the link periods, are the ramification indices in fibers above points on the $i$ th boundary component of $X$. Associated with each signature is a presentation for the NEC group $\Gamma$. For these presentations and more information about signatures, see [7] and [13].

Glasgow Math. J. 33 (1991) 61-71. 
Let $\Gamma$ be an NEC group with signature (2.1). Then the non-euclidean area $\mu(\Gamma)$ of a fundamental region for $\Gamma$ can be calculated directly from the signature $[13$, p. 235]:

$$
\mu(\Gamma) / 2 \pi=\alpha p-2+\sum_{i=1}^{r}\left(1-\frac{1}{m_{i}}\right)+k+\sum_{i=1}^{k} \sum_{j=1}^{s_{i}} \frac{1}{2}\left(1-\frac{1}{n_{i j}}\right),
$$

where $\alpha=2$ if $D / \Gamma$ is orientable and $\alpha=1$ if $D / \Gamma$ is non-orientable. If $\Delta$ is a subgroup of finite index in $\Gamma$, then $\Delta$ is an NEC group and

$$
[\Gamma: \Delta]=\mu(\Delta) / \mu(\Gamma) \text {. }
$$

There is a collection of results $[3$, p. 506] that can sometimes be applied to determine the signature of $\Delta$ from that of $\Gamma$.

An NEC group $K$ is called a surface group if the quotient map from $D$ to $D / K$ is unramified. Fuchsian surface groups contain no elements of finite order. If the quotient space $D / K$ has a non-empty boundary, then $K$ is called a bordered surface group. Bordered surface groups contain reflections but no other elements of finite order.

Let $X$ be a compact Klein surface of algebraic genus $g \geq 2$. Then $X$ can be represented as $D / K$ where $K$ is a surface group. If $X$ is a classical Riemann surface, then $K$ is a Fuchsian group, and if $X$ is a Klein surface with non-empty boundary, then $K$ is a bordered surface group.

The full automorphism group $A(X)$ is isomorphic to $N(K) / K$ where $N(K)$ is the normalizer of $K$ in $\mathscr{L}[8, \mathrm{p}$. 4]. If $X$ is a Riemann surface so that the Fuchsian group $K \subset \mathscr{L}^{+}$, then the group $A^{+}(X)$ of orientation-preserving automorphisms of $X$ is isomorphic to $N^{+}(K) / K$, where $N^{+}(K)$ is the normalizer of $K$ in $\mathscr{L}^{+}$.

Especially important in the study of automorphisms of Riemann surfaces are the triangle groups. A triangle group is a Fuchsian group with signature

where

$$
(0 ;+;[l, m, n] ;\{\})
$$

$$
1 / l+1 / m+1 / n<1 \text {. }
$$

We shall denote a group with this signature by $\Gamma(l, m, n)$. The extended triangle group $\Gamma[l, m, n]$ is a proper NEC group with signature

$$
(0 ;+;[] ;\{(l, m, n)\}) \text {. }
$$

Its canonical Fuchsian group is a triangle group $\Gamma(l, m, n)$. Large groups of orientationpreserving automorphisms are quotients of triangle groups. Singerman made this idea precise in the following [15, p. 22].

LEMMA A. Let $G$ be a group of orientation-preserving automorphisms of a Riemann surface of genus $g \geq 2$. If $o(G)>12(g-1)$, then $G$ is a quotient of a triangle group. If $o(G)>24(g-1)$, then, further, one of the periods of the triangle group is 2 .

Large groups of automorphisms of bordered Klein surfaces are quotients of proper NEC groups. A group that acts as the automorphism group of a bordered surface with maximal symmetry is called an $M^{*}$-group [8]. The first important result about $M^{*}$-groups was that they must have a certain partial presentation $\left[8\right.$, p. 5]. An $M^{*}$-group $G$ is generated by three distinct non-trivial elements $T, U$, and $V$ which satisfy the relations

$$
T^{2}=U^{2}=V^{2}=(T U)^{2}=(T V)^{3}=1 .
$$


This was established using NEC groups. The finite group $G$ is the automorphism group of a bordered surface $X$ with maximal symmetry if and only if there are an NEC group $\Delta$ with signature

$$
(0 ;+;[] ;\{(2,2,2,3)\})
$$

and a homomorphism $\alpha: \Delta \rightarrow G$ onto $G$ such that $X=D / K$ where $K=$ kernel $\alpha$ is a bordered surface group [8, pp. 4-6]. There is a similar result about groups of the second largest possible order.

The order of $U V$ is called an index of the $M^{*}$-group $G$ [6], and there is a nice connection between the index and the action of $G$ on $X$ [6, p. 282]. If $X$ has $k$ boundary components and $q=o(U V)$, then $o(G)=2 q k$.

3. Complex doubles. Let $X$ be a bordered Klein surface. Associated with $X$ in a very natural way is its complex double $X_{c}$ [1, pp. 37-41], a Riemann surface of the same genus. The surface $X_{c}$ has an antianalytic involution $\sigma: X_{c} \rightarrow X_{c}$ such that $X_{c} / \sigma=X$. The automorphism groups of $X$ and $X_{c}$ are intimately connected [1, p. 79]:

$$
A(X) \cong\left\{f \in A^{+}\left(X_{c}\right) \mid \text { of }=f \sigma\right\} .
$$

Now let $X$ be a bordered Klein surface with maximal symmetry of genus $g$, and let $H=A(X)$, an $M^{*}$-group. There is an NEC group $\Delta$ with signature (2.5) and a homomorphism $\alpha: \Delta \rightarrow H$ onto $H$ such that $X=D / K$ where $K=$ kernel $\alpha$ is a bordered surface group. The surface $D / K^{+}$is the complex double of $X[8, \mathrm{p} .3]$, and we have the following lattice of subgroups of $\mathscr{L}$.

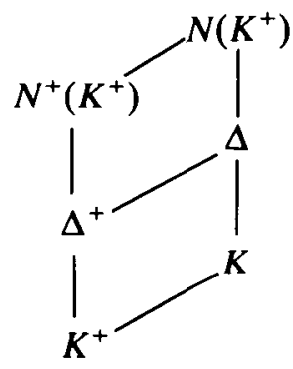

Here $\Delta^{+} / K^{+} \cong \Delta / K \cong H, K / K^{+} \cong\langle\sigma\rangle$, and $\Delta / K^{+} \cong\langle\sigma\rangle \times H$.

Now let $G=A^{+}\left(X_{c}\right)$. Then $H$ is isomorphic to a subgroup of $G$ by (3.1), and $\langle\sigma\rangle \times H$ is isomorphic to the centralizer of $\sigma$ in $A\left(X_{c}\right)$. Since $X$ has maximal symmetry, $o(H)=12(g-1)$ and thus $o(G)$ is a multiple of $12(g-1)$. There are very few possibilities for $o(G)$. We have the following easy result.

Proposition 1. Let $W$ be a Riemann surface of genus $g \geq 2$, and let $G=A^{+}(W)$. If $W$ is the complex double of a bordered surface with maximal symmetry, then $o(G)$ is a multiple of $12(g-1)$. If further $o(G)>24(g-1)$, then $o(G)$ is one of the following; in each case $G$ is a quotient of the triangle group listed.

$$
\begin{array}{ll}
o(G)=84(g-1) & \Gamma(2,3,7) \\
o(G)=48(g-1) & \Gamma(2,3,8) \\
o(G)=36(g-1) & \Gamma(2,3,9)
\end{array}
$$


If $o(G)=24(g-1)$, then $G$ is a quotient of $\Gamma(2,4,6), \Gamma(2,3,12)$ or $\Gamma(3,3,4)$. If $o(G)=12(g-1)$, then $G$ is a quotient of a Fuchsian group with signature

$$
(0 ;+;[2,2,2,3] ;\{\}) \text {. }
$$

In any case the order of the full automorphism group $A(W)$ is twice the order of $G$.

Proof. Let $X$ be the bordered surface with maximal symmetry and use the notation in the diagram (3.2). First suppose $o(G)=12(g-1)$. Then, simply, the full group $A(W) \cong \Delta / K^{+}$and $G \cong \Delta^{+} / K^{+}$. The signature of the canonical Fuchsian subgroup $\Delta^{+}$is (3.3) $[13$, p. 236].

Next suppose $o(G)>12(g-1)$. By Lemma $\mathrm{A}, G$ is a quotient of a triangle group $\Gamma=\Gamma(l, m, n)$, and we may take $l \leq m \leq n$. Then $G \cong \Gamma / K^{+}$, where $\Gamma=N^{+}\left(K^{+}\right)$. From (2.2) $\mu\left(K^{+}\right)=4 \pi(g-1)$ and $\mu(\Gamma)=2 \pi(1-1 / l-1 / m-1 / n)$. Then $o(G)=\mu\left(K^{+}\right) / \mu(\Gamma)$ by (2.3), and $o(G)$ is a multiple of $12(g-1)$. It is now a simple matter to check all possibilities for $l, m$ and $n$.

4. Surfaces of low genus. Here we examine the large automorphism groups of Riemann surfaces of genus $g$, where $2 \leq g \leq 5$. The proof of our main result depends upon this study of the low genera, although perhaps the examples help illuminate the theoretical development.

Let $W$ be a Riemann surface of genus $g, 2 \leq g \leq 5$. First we determine the possibilities for $A^{+}(W)$ with order equal to $36(g-1), 48(g-1)$ or $84(g-1)$; fortunately there are not many. For each possible group we next obtain a presentation for $A(W)$ as a quotient of the appropriate extended triangle group; Theorem 2 of [15] is helpful when only the presentation for $A^{+}(W)$ is known. Then for each reflection $\tau$ in $A(W)$, we calculate the order of its centralizer $C(\tau)$ in $A(W)$. This can be determined by finding the number of conjugates of $\tau$ in $A(W)$, since this number equals the index of $C(\tau)$ in $A(W)$. If the order of $C(\tau)$ is less than $24(g-1)$, then the bordered Klein surface $W / \tau$ does not have maximal symmetry. It turns out that in these genera, no Riemann surface with more than $24(g-1)$ orientation-preserving automorphisms is the complex double of a surface with maximal symmetry. We omit the details.

Proposition 2. Let $X$ be a bordered Klein surface with maximal symmetry of genus $g$, $2 \leq g \leq 5$. Then the order of $A\left(X_{c}\right)$ is $24(g-1)$ or $48(g-1)$.

There is a strong connection here with the theory of regular maps on surfaces. We are using "regular" in the usual way [4], not in the strong sense of [6]. Large groups of automorphisms of Riemann surfaces correspond to groups of regular maps. A Riemann surface is called symmetric [15] if it has an anti-conformal involution.

Let $G$ be a group of conformal automorphisms of a Riemann surface $W$. If $G$ is a quotient of a triangle group $\Gamma(2, n, k)$, then there is a regular map of type $\{n, k\}$ on the topological surface $W$. If the map is reflexible, then $W$ is a symmetric Riemann surface, and further $A(W)$ is isomorphic to the full automorphism group of the map.

Conversely, if $G$ is the rotation group of a regular map of type $\{n, k\}$ on a surface $S$, then $G$ is a quotient of a triangle group $\Gamma(2, n, k)$ and $G$ acts as a group of conformal automorphisms of a Riemann surface homeomorphic to $S$. However, the symmetry of the Riemann surface need not imply the reflexibility of the map. Indeed there are symmetric 
Riemann surfaces that correspond to irreflexible maps [15, p. 30]. However, irreflexible maps of positive genus are rather exceptional, and in fact Garbe [5, p. 42] has shown that for $2 \leq g \leq 6$, there are no irreflexible maps at all. This is relevant here. Also, if $n \neq k$, then the symmetry of the Riemann surface does imply the reflexibility of the map. For more details on this correspondence, see [15, pp. 27, 28].

Hence, for $2 \leq g \leq 5$, symmetric Riemann surfaces with large automorphism groups correspond to reflexible regular maps. A great deal is known about the automorphism groups of Riemann surfaces and regular maps of these genera. The possibilities for $A^{+}(W)$ when $W$ has genus 2 were first determined by Wiman in 1895 , and the full automorphism groups are worked out in [3]. The regular maps of genus 3 were classified by Sherk [12], and those of genera 4 and 5 by Garbe [5]. The possibilities that must be considered to establish Proposition 2 are in the table. The symbols for the maps and groups are from [4].

\begin{tabular}{ccccrcc}
\hline Genus & Map & $A^{+}(W)$ & $A(W)$ & $\begin{array}{c}\text { Order } \\
A(W)\end{array}$ & $\begin{array}{c}\text { Triangle } \\
\text { group }\end{array}$ & References \\
\hline 2 & $\{3,4+4\}$ & $\langle-3,4 \mid 2\rangle$ & & 96 & $\Gamma(2,3,8)$ & {$[\mathbf{4}$, p. 140], [12, p. 460], [3, p. 518] } \\
3 & $\{3,8\}_{6}$ & $(2,3,8 ; 3)$ & $G^{3.8 .6}$ & 192 & $\Gamma(2,3,8)$ & {$[\mathbf{4}$, p. 139], [12, p. 475] } \\
3 & $\{3,8\}_{7}$ & PSL(2,7) & PGL(2,7) & 336 & $\Gamma(2,3,7)$ & {$[\mathbf{4}$, p. 139], [12, p. 475] } \\
5 & & & & 384 & $\Gamma(2,3,8)$ & {$[\mathbf{5 , p . 5 4 ]}$} \\
\hline
\end{tabular}

We conclude this section by presenting the Klein surface with maximal symmetry such that its complex double has $48(g-1)$ automorphisms.

EXAMPLE. Let $G_{48}$ be the group with generators $A, B$, and $C$ and defining relations

$$
A^{2}=B^{2}=C^{2}=(A B)^{4}=(B C)^{6}=(A C)^{2}=(B A B C)^{2}=1 \text {. }
$$

The group $G_{48}$ is a group of order 48 that acts on a unique Riemann surface $W$ of genus two [3, p. 517]. Also $G_{48}$ is the full automorphism group of the map $\{4,6 \mid 2\}[4, p .110]$, the only regular map of type $\{4,6\}$ with genus two. The group $G_{48}$ is a quotient of the extended triangle group $\Gamma[2,4,6]$, and the uniqueness of $W$ follows from the uniqueness of this triangle group together with the classification of the regular maps of genus two [4, p. 140]. Also see [3, p. 518]. The centralizer of the reflection $A$ has order 24 , so that the Klein surface $Y=W / A$ has the maximal symmetry. Topologically $Y$ is a sphere with three holes; the automorphism group of $Y$ is $D_{6}$, the only $M^{*}$-group of genus two.

The conjugacy class of $A$ is $\{A, B A B\}$, and the Klein surfaces $W / A$ and $W / B A B$ are dianalytically equivalent $[1, \mathrm{pp} .57,58]$. Outside this conjugacy class no reflection has a centralizer with order greater than 16. Thus there is a unique bordered Klein surface $Y$ of genus two with maximal symmetry and complex double $W$. Theorem 1 says that $Y$ is very special indeed.

5. The proof of Theorem 1-part one. We now show that the automorphism group of the complex double of a surface with maximal symmetry cannot be too large. We first eliminate the three largest possible group orders in Proposition 1.

Let $X$ be a bordered Klein surface with maximal symmetry of genus $g \geq 2$, and let $W$ 
be its complex double. Let $\sigma$ be the antianalytic involution of $W$ such that $W / \sigma=X$. We set

$$
G=A^{+}(W) \quad H=\{f \in G \mid \sigma f=f \sigma\} \quad L=\langle\sigma\rangle
$$

Then $H$ is isomorphic to the $M^{*}$-group $A(X)=(L \times H) / L$. We identify $H$ and $A(X)$.

The following is basic.

Lemma 1. Suppose $N$ is a normal subgroup of $G$ such that $N \subset H$ with $[H: N]>6$. Set $W^{\prime}=W / N, X^{\prime}=X / N, G^{\prime}=G / N, H^{\prime}=H / N$. Then

1) $X^{\prime}$ is a bordered surface with maximal symmetry of genus $g^{\prime} \geq 2$.

2) $W^{\prime}$ is the complex double of $X^{\prime}$.

3) The following diagram commutes, and each quotient mapping is unramified.

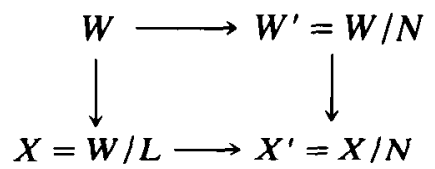

Proof. Part 1) was established in [6, p. 271]. We are identifying $N$ and $(L \times N) / L$, so that $X^{\prime}=X /((L \times N) / L)=W /(L \times N)=(W / N) /((L \times N) / N)$. Thus the diagram commutes, and $(L \times N) / N \cong C_{2}$ acts on $W^{\prime}$ as a reflection. The quotient space $W^{\prime}$ is a Riemann surface since $N \subset A^{+}(W)$. The mapping $X \rightarrow X^{\prime}$ is unramified [6, p. 271] so that each mapping in the diagram must be unramified. Finally $W^{\prime}$ is the complex double of $X^{\prime}$, by the uniqueness of this covering [1, p. 37].

Corollary 1. If $o(G)=k(g-1)$, then $o\left(G^{\prime}\right)=k\left(g^{\prime}-1\right)$.

Proof. Since the quotient map from $W$ to $W^{\prime}$ is unramified, the classical Hurwitz ramification formula gives

$$
g-1=o(N) \cdot\left(g^{\prime}-1\right) .
$$

CoRollary 2. If $o(G)=k(g-1)$ where $k$ is 36,48 , or 84 , then $G^{\prime}=A^{+}\left(W^{\prime}\right)$.

Proof. Since $G^{\prime}$ is a subgroup of $A^{+}\left(W^{\prime}\right)$, the result follows by Lagrange's Theorem. We also need the following technical result.

Lemma 2. Let $H$ be a solvable $M^{*}$-group with $o(H) \geq 48$. Suppose $A$ is a normal subgroup with $[H: A]=6$. If $A$ is an elementary abelian 2-group, then $H \cong C_{2} \times S_{4}$.

Proof. The quotient $H / A \cong S_{3}$, the only possible quotient of an $M^{*}$-group of order 6. Now it is not hard to see that $H$ has no elements with order larger than 6 . Hence $H$ is a quotient of a group $G^{3, m, n}$, where $m \leq n \leq 6[6, \mathrm{p} .278]$. Then from the table in [4, p. 139], the only possibility is $H \cong C_{2} \times S_{4}$.

The following result gives a way to find normal subgroups with index larger than 6 in solvable $M^{*}$-groups. As usual, we denote the Frattini subgroup of a finite group $G$ by $\Phi(G)$. Also, if $p$ is a prime, then $G$ has a unique largest normal $p$-subgroup [10, p. 57], which is denoted $O_{p}(G)$. Both $\Phi(G)$ and $O_{p}(G)$ are characteristic subgroups of $G$.

Lemma 3. Let $H$ be a solvable $M^{*}$-group with $o(H)>48$, and let $N$ be a normal subgroup of $H$ with $[H: N]=6$. Then $N$ has a nontrivial characteristic subgroup $C$. 
Proof. Since $N$ is solvable, $O_{p}(N) \neq 1$ for some prime $p\left[10\right.$, p. 155]. If $O_{p}(N) \neq N$, then set $C=O_{p}(N)$. So assume $O_{p}(N)=N$. Since 12 divides $o(H)$, we must have $p=2$ and $N$ is a 2-group. Now $\Phi(N) \neq N$. If $\Phi(N)=1$, then $N$ would be an elementary abelian 2-group. But this would contradict Lemma 2. Hence $\Phi(N) \neq 1$. Now set $C=\Phi(N)$.

Proposition 3. The order of $G$ is not $48(g-1)$.

Proof. Suppose to the contrary that there are bordered surfaces with maximal symmetry that have complex doubles with $48(g-1)$ orientation-preserving automorphisms. Assume that $X$ is such a bordered surface of the lowest genus. By Proposition 2, we know its genus $g>5$.

Now $o(G)=48(g-1)$ so that $G$ is a quotient of a $\Gamma(2,3,8)$ triangle group. Thus $G$ is solvable [11, p. 19].

Also $[G: H]=4$ and there is a homomorphism $f: G \rightarrow S_{4}$ with $J=\operatorname{kernel} f \subset H$. Since $H$ is an $M^{*}$-group, the order of $H / J$ cannot be 3 . Thus $[H: J]=1,2$, or 6 . In each case $J$ has a characteristic subgroup $C$ with $[H: C]>6$. If $[H: J]=6$, then this is immediate from Lemma 3. The other two cases are harder.

First assume $[H: J]=2$. Clearly $H^{\prime} \subset J$, and we know $\left[H: H^{\prime}\right]$ divides 4 [6, p. 278]. Hence either $H^{\prime}=J$ or $\left[J: H^{\prime}\right]=2$.

Suppose $H^{\prime}=J$. Then $H^{\prime \prime}=J^{\prime}$ of course. But $\left[H^{\prime}: H^{\prime \prime}\right]$ divides $9[6$, p. 278]. The group $H^{\prime}$ is solvable, and a quotient of the $M^{*}$-group $H$ cannot have order 18 . Hence $\left[H^{\prime}: H^{\prime \prime}\right]=3$. Now $\left[H: H^{\prime \prime}\right]=6$, and by Lemma $3 H^{\prime \prime}$ has nontrivial characteristic subgroup $C$. Since $H^{\prime \prime}=J^{\prime}$ is characteristic in $J$, so is $C$.

Next suppose $\left[J: H^{\prime}\right]=2$. Then $H^{\prime \prime} \subset J^{\prime} \subset H^{\prime}$ and $\left[H^{\prime}: H^{\prime \prime}\right]$ is 3 or 9 . If $J^{\prime}=H^{\prime}$, then $H^{\prime \prime}$ is characteristic in $J$ and $\left[H: H^{\prime \prime}\right]$ is 12 or 36 ; set $C=H^{\prime \prime}$. If $J^{\prime} \neq H^{\prime}$, then $\left[H^{\prime}: J^{\prime}\right] \geq 3$ and $\left[H: J^{\prime}\right] \geq 12$; set $C=J^{\prime}$.

Now assume $H=J$. Then $\left[H: H^{\prime \prime}\right] \geq 6$. If $\left[H: H^{\prime \prime}\right]>6$, then take $C=H^{\prime \prime}$. If $\left[H: H^{\prime \prime}\right]=6$, then $H^{\prime \prime}$ has a nontrivial characteristic subgroup $C$ by Lemma 3 again, and $C$ is characteristic in $J$.

Since $J$ is normal in $G$, so is its characteristic subgroup $C$ [10, p. 40]. Now applying Lemma 1 produces a bordered surface $X^{\prime}=X / C$ of lower genus $g^{\prime}$ that has maximal symmetry and a complex double with $48\left(g^{\prime}-1\right)$ orientation-preserving automorphisms. But this contradicts the choice of $X$.

The proof of the following is similar but easier, and we omit the details.

Proposition 4. The order of $G$ is not $36(g-1)$.

The key here is that if $o(G)$ were $36(g-1)$, then $G$ would be a quotient of a $\Gamma(2,3,9)$ triangle group and therefore solvable [11, p. 19]. different.

Since the $\Gamma(2,3,7)$ triangle group is not solvable, the proof in the remaining case is

Proposition 5. The order of $G$ is not $84(g-1)$.

Proof. Suppose to the contrary that $o(G)=84(g-1)$. Now $[G: H]=7$ and there is a homomorphism $f: G \rightarrow S_{7}$ with $J=$ kernel $f \subset H$. Now $o(G / J)$ divides $7 !=84.60$. But $G / J$ is a Hurwitz group. Let $g^{\prime}$ be the genus of $W / J$ so that $G / J$ has order $84\left(g^{\prime}-1\right)$. Now $g^{\prime}-1$ divides 60 . The Hurwitz groups of low order are well known [11, pp. 37, 38]. The only possibilities for $g^{\prime}$ are 7 and 3 . But $H / J$ is an $M^{*}$-group. Since there are no $M^{*}$-groups of genus 7 [9, p. 392], we must have $g^{\prime}=3$. But this contradicts Proposition 2 . 
6. The proof of Theorem 1-part two. Here we consider the remaining possibilities in Proposition 1. The approach of $\$ 5$ will not work here due to the existence of the surface $Y$ of genus two. We use NEC groups.

Let $X$ be a bordered Klein surface of genus $g \geq 2$, and suppose the $M^{*}$-group $H$ acts on $X$. Then represent $X$ as $D / K$ where $K$ is a bordered surface group. Then there is an NEC group $\Delta$ with signature (2.5) such that $H=\Delta / K$.

Now let $G=A^{+}\left(X_{c}\right), G^{*}=A\left(X_{c}\right)$ and assume $o(G)=24(g-1)$. We have the subgroup lattice (3.2), but now $\Delta^{+}$is a normal subgroup of index 2 in $N^{+}\left(K^{+}\right)$. The normality is quite helpful. Of course $G \cong N^{+}\left(K^{+}\right) / K^{+}$.

Proposition 6. The full automorphism group $G^{*}$ is a quotient of the extended triangle group $\Gamma[2,4,6]$.

Proof. By Proposition $1, N^{+}\left(K^{+}\right)$is either $\Gamma(2,4,6), \Gamma(2,3,12)$ or $\Gamma(3,3,4)$. It is easy to see that neither $\Gamma(2,3,12)$ nor $\Gamma(3,3,4)$ contains a subgroup isomorphic to $\Delta^{+}$.

First let $\Gamma=\Gamma(2,3,12)$ have presentation

$$
x^{3}=y^{12}=(x y)^{2}=1 .
$$

Assume $\Lambda$ is a subgroup of $\Gamma$ with $[\Gamma: \Lambda]=2$. Then $x, y^{2} \in \Lambda, y \notin \Lambda$ so that $y^{2}$ induces an ordinary period 6 on $\Lambda[3$, p. 506]. Thus $\Lambda$ could not have signature (3.3).

The group $\Gamma(3,3,4)$ is generated by two elements of order three and thus has no subgroups of index two.

Therefore, $N^{+}\left(K^{+}\right)$is the triangle group $\Gamma(2,4,6)$, and $N\left(K^{+}\right)$is $\Gamma[2,4,6]$, since there is no other NEC group with its canonical Fuchsian subgroup isomorphic to $\Gamma(2,4,6)$ $[15$, p. 21].

Now let $\Gamma^{*}=\Gamma[2,4,6]$ have presentation

$$
a^{2}=b^{2}=c^{2}=(a b)^{4}=(b c)^{6}=(a c)^{2}=1 .
$$

We have the following diagram of groups and quotient mappings.

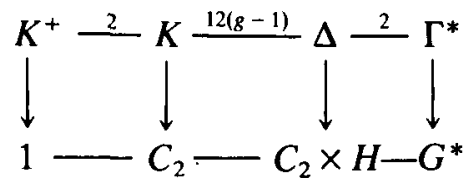

There is only one possibility for $\Delta$.

Proposition 7. $\Delta=\langle a, c, b a b, b c b\rangle$.

Proof. The group $\Delta$ is a normal subgroup of index two in $\Gamma^{*}$. If any two of the three generators $a, b, c$ were not in $\Delta$, then the product of these two would be in $\Delta$ and $\Delta$ would have an ordinary period $[3$, p. 506]. Hence exactly one of the three generators is not in $\Delta$. If $a \notin \Delta$, then $\Delta$ would have a link period 6 induced by $b, c[3$, p. 506]. If $c \notin \Delta$, then $a, b$ would induce a link period 4 on $\Delta$. Therefore $b \notin \Delta, a, c \in \Delta$. Also $b a b, b c b \in \Delta$ since $\Delta$ is normal in $\Gamma^{*}$.

Now let $M=\langle a, c, b a b, b c b\rangle$. Then clearly $M \subset \Delta, M$ is normal in $\Gamma^{*}$ and $\Gamma^{*} / M \cong C_{2}$. Hence $\Delta=M$.

Now set $t=b c b, u=b a b, j=a$, and $v=c$. The four elements $t, u, j$ and $v$ generate 
$\Delta$, of course, and it is a simple matter to check that they satisfy the relations

$$
t^{2}=u^{2}=j^{2}=v^{2}=(t u)^{2}=(u j)^{2}=(j v)^{2}=(t v)^{3}=1 .
$$

Thus $t, u, j$ and $v$ form a standard generating set for the group $\Delta$. Now consider the quotient mapping $\phi: \Delta \rightarrow H$ of $\Delta$ onto the $M^{*}$-group $H$. Following the proof of Theorem 1 of [8], we see that $t \notin K=\operatorname{kernel} \phi, v \notin K$, but one of the remaining two generators is in $K$.

Assume $j \in K$. The proof in the other case is very similar. Let $T=\phi(t), U=\phi(u)$, and $V=\phi(v)$. Then $T, U$ and $V$ generate the $M^{*}$-group $H$ and satisfy the relations (2.4). (If $u \in K$, then choose $T=\phi(v), U=\phi(j), V=\phi(t)$.) See [8, pp. 5, 6].

Next consider the quotient mapping $\alpha: \Delta \rightarrow C_{2} \times H$ of $\Delta$ onto $C_{2} \times H$ with kernel $\alpha=K^{+}$. Since the reflection $j$ is in $K$ but not in the Fuchsian surface group $K^{+}$, the image $\alpha(j)$ must generate the factor $C_{2}$. Write $J=\alpha(j)$. Then the direct product $C_{2} \times H$ has generators $J, T, U$ and $V$ that satisfy the relations (2.4) and

$$
J^{2}=(J T)^{2}=(J U)^{2}=(J V)^{2}=1 .
$$

The reflection $J$ acts on the complex double $X_{c}=D / K^{+}$and $X=X_{c} / J$.

But conjugation by $b$ is an inner automorphism $h$ of $\Gamma^{*}$ that interchanges $t$ and $v$ and also $u$ and $j$. This is perhaps the crucial observation. The normal subgroup $K^{+}$is invariant under $h$, of course. Therefore $h$ induces an automorphism $\theta$ of the quotient group $G^{*}=\Gamma^{*} / K^{+}$. This is severely limiting, since $J$ generates the factor $C_{2}$ of $C_{2} \times H$.

Proposition 8. The index of the $M^{*}$-group $H$ is $2, H \cong D_{6}$ and the genus of the bordered Klein surface $X$ is 2. Topologically $X$ is a sphere with three holes.

Proof. The index $q$ of $H$ is $o(U V)$. The action of $\theta$ on $G^{*}$ is to interchange $T$ and $V$ and also $U$ and $J$. But the order of $U V$ is equal to the order of $\theta(U V)=J T$, which is two. Hence $q=2$ and $H \cong D_{6}$, the only $M^{*}$-group with index 2 [9, p. 377]. With this index, $D_{6}$ acts on a unique topological type of bordered surface, a sphere with three holes.

It is interesting that the index of the $M^{*}$-group must be two. In fact, we have established more.

Proposition 9. $G^{*} \cong G_{48}$, and $X$ is dianalytically equivalent to the surface $Y$ of genus two.

Proof. We have $o\left(G^{*}\right)=48$. Let $\pi: \Gamma^{*} \rightarrow G^{*}$ be the quotient mapping, and write $A=\pi(a), B=\pi(b), C=\pi(c)$. In $G^{*},(U V)^{2}=(B A B . C)^{2}=1$, from the previous proof. Thus the generators $A, B$ and $C$ for $G^{*}$ satisfy the defining relations (4.1) for $G_{48}$. Hence $G^{*} \cong G_{48}$.

Now the complex double $X_{c}$ is the Riemann surface $W$ of the example of $\S 4$ and $X$ is the surface $Y=W / A$, since $A=J$.

Note that the other possibility for kernel $\phi$ gives the surface $W / B A B$. This concludes the proof of Theorem 1.

7. Teichmuller space. Let $\Gamma$ be a NEC group, and let $T(\Gamma)$ be the Teichmuller space of $\Gamma$. Then $T(\Gamma)$ is homeomorphic to a real open ball of dimension $d(\Gamma)$. If $\Gamma$ is a Fuchsian group with signature $\left(g ;+;\left[m_{1}, \ldots, m_{r}\right] ;\{\}\right)$, then $d(\Gamma)=6 g-6+2 r$. If $\Gamma$ is a proper NEC group, then $d(\Gamma)=d\left(\Gamma^{+}\right) / 2[15$, p. 19]. 
Now let $\Gamma_{1}$ and $\Gamma_{2}$ be NEC groups, and let $\alpha: \Gamma_{1} \rightarrow \Gamma_{2}$ be a monomorphism. Then $\alpha$ induces an embedding $\alpha_{*}: T\left(\Gamma_{2}\right) \rightarrow T\left(\Gamma_{1}\right)$. The points in the image of this embedding correspond to groups isomorphic to $\Gamma_{1}$ which are contained in groups isomorphic to $\Gamma_{2}$. In particular, if $K$ is a surface group that is a normal subgroup of $\Gamma$, then the points in the image of the embedding of $T(\Gamma)$ in $T(K)$ correspond to surfaces with a group of automorphisms isomorphic to $\Gamma / K$.

An NEC group $\Gamma_{1}$ is said to be maximal if $\Gamma_{1}$ is not a proper subgroup of another NEC group. Let $\operatorname{Max}\left(\Gamma_{1}\right)$ denote the subset of $T\left(\Gamma_{1}\right)$ that consists of the maximal groups. Then $\operatorname{Max}\left(\Gamma_{1}\right)$ is usually an open, everywhere dense subset of $T\left(\Gamma_{1}\right)$. However there are some exceptional NEC groups for which $\operatorname{Max}\left(\Gamma_{1}\right)$ is empty.

The signatures of all Fuchsian groups for which $\operatorname{Max}\left(\Gamma_{1}\right)$ is empty have been classified by Singerman [14]. In addition, Bujalance [2] has determined all inclusions between NEC groups with $d\left(\Gamma_{1}\right)=d\left(\Gamma_{2}\right)$, with the additional condition that $\Gamma_{1}$ is a normal subgroup of $\Gamma_{2}$.

Now let $X$ be a bordered Klein surface with maximal symmetry, and let $H=A(X)$. Then there are an NEC group $\Delta$ with signature (2.5) and a homomorphism $\phi: \Delta \rightarrow H$ onto $\dot{H}$ such that $X=D / K$ where $K=\operatorname{kernel} \phi$ is a bordered surface group. We have the inclusions

$$
K^{+}-K-\Delta
$$

and induced imbeddings of the Teichmuller spaces

$$
T(\Delta)-T(K)-T\left(K^{+}\right) \text {. }
$$

The points in the image of $T(\Delta)$ in $T(K)$ correspond to bordered Klein surfaces with automorphism group $H$; each surface has the same topological type as $X$. The points in the image of $T(\Delta)$ in $T\left(K^{+}\right)$correspond to Riemann surfaces with a group of automorphisms isomorphic to $C_{2} \times H$; each surface is the complex double of a bordered Klein surface with maximal symmetry. Now let $F$ denote the set of points in the image of $T(\Delta)$ in $T\left(K^{+}\right)$that correspond to Riemann surfaces with full automorphism group $C_{2} \times H$. The set $F$ is just the image of $\operatorname{Max}(\Delta)$. The canonical Fuchsian group $\Delta^{+}$has signature (3.3), and this signature is not one of the exceptional ones [14, p. 33]. Therefore $\Delta$ is not exceptional either, and $F$ is an open, everywhere dense subset of the image of $T(\Delta)$ in $T\left(K^{+}\right)$. Just from these considerations, then, it follows that for most Klein surfaces with the same topological type as $X$ and automorphism group $H$, the complex double of the surface has full automorphism group $C_{2} \times H$. Theorem 1 says that, except for one topological type, all the surfaces have complex doubles with that automorphism group. Most spheres with three holes that have maximal symmetry have complex doubles with automorphism group $C_{2} \times D_{6}$. However, the complex double of the special surface $Y$ has automorphism group $G_{48}$, which is twice as big.

There is also a connection here with the main result of [3], which classifies the symmetry types of Riemann surfaces of genus two. There must be Riemann surfaces of genus two with automorphism group $C_{2} \times D_{6}$ that are complex doubles of orientable surfaces with three boundary components, but no surface with species +3 appears in the table [3, p. 518]. However, Singerman has informed us [16] that the table should have an additional entry, a surface $X$ with $A^{+}(X)=D_{6}, A(X)=C_{2} \times D_{6}$, UCT group $(0 ;+$; $[2,2,2,3] ;\{\})$, EUCT group $(0 ;+;[] ;\{(2,2,2,3)\})$ and symmetry type $\{0,+1,+3,+3\}$. 
Finally, we would like to thank David Singerman for his help with the ideas of $\$ 6$ and $\$ 7$.

\section{REFERENCES}

1. N. L. Alling and N. Greenleaf, Foundations of the theory of Klein surfaces, Lecture Notes in Mathematics Vol. 219 (Springer-Verlag, 1971).

2. E. Bujalance, Normal N.E.C. signatures, Illinois J. Math. 26 (1982), 519-530.

3. E. Bujalance and D. Singerman, The symmetry type of a Riemann surface, Proc. London Math. Soc. (3) 51 (1985), 501-519.

4. H. S. M. Coxeter and W. O. J. Moser, Generators and relations for discrete groups, 3rd ed., Ergebnisse der Mathematik und ihrer Grenzgebiete Band 14 (Springer-Verlag, 1972).

5. D. Garbe, Über die regularen Zerlegungen geschlossener orientierbarer Flachen, J. Reine Angew. Math. 237 (1969), 39-55.

6. N. Greenleaf and C. L. May, Bordered Klein surfaces with maximal symmetry, Trans. Amer. Math. Soc. 274 (1982), 265-283.

7. A. M. Macbeath, The classification of non-Euclidean plane crystallographic groups, Canad. J. Math. 19 (1966), 1192-1205.

8. C. L. May, Large automorphism groups of compact Klein surfaces with boundary, Glasgow Math. J. 18 (1977), 1-10.

9. C. L. May, The species of bordered Klein surfaces with maximal symmetry of low genus, Pacific J. Math. 111 (1984), 371-394.

10. J. S. Rose, $A$ course on group theory (Cambridge University Press, 1978).

11. C. H. Sah, Groups related to compact Riemann surfaces, Acta Math. 123 (1969), 13-42. 452-480.

12. F. A. Sherk, The regular maps on a surface of genus three, Canad. J. Math. 11 (1959),

13. D. Singerman, On the structure of non-Euclidean crystallographic groups, Proc. Cambridge Philos. Soc. 76 (1974), 233-240. $29-38$

14. D. Singerman, Finitely maximal Fuchsian groups, J. London Math. Soc. (2) 6 (1972),

15. D. Singerman, Symmetries of Riemann surfaces with large automorphism group, Math. Ann. 210 (1974), 17-32.

16. D. Singerman, private communication, 1988.

\section{Department of Mathematics}

TOWSON STATE UnIVERSITY

TOWSON

MARYLAND 21204-7097

USA 